\title{
Short communication: Characterization of gene expression profiles related to yak milk protein synthesis during the lactation cycle
}

\author{
Wei Xia, ${ }^{*} \dagger$ Johan S. Osorio,‡ Yuanxiao Yang, ${ }^{*} \dagger$ DongLiang Liu, $\S$ and Ming Feng Jiang ${ }^{*} \dagger^{1}$ \\ ${ }^{*}$ College of Life Science and Technology, Southwest Minzu University, Chengdu 610041, China \\ †Key Laboratory of Qinghai-Tibetan Plateau Animal Genetic Resource Conservation and Exploitation, Key Laboratory of Animal Genetics \\ \& Breeding of State Ethnic Affairs Commission and Ministry of Education, Southwest University for Nationalities, Chengdu 610041, China \\ ‡Dairy and Food Science Department, South Dakota State University, Brookings 57007 \\ $\S 363$ Hospital, Chengdu 610041, China
}

\section{ABSTRACT}

This research assessed the gene expression patterns related to the synthesis of milk in yak, which is characterized by high fat and protein content but low yield. The yak (Bos grunniens) is one of the most crucial domestic animals in Tibetan life; however, the genetic and molecular factors underlying yak milk protein synthesis remain understudied. Yak mammary biopsies harvested during late-pregnancy $(\mathrm{d}-15)$ through the end of subsequent lactation (d 1, 15, 30, 60, 180, and 240 ) were used to evaluate gene expression via real-time quantitative PCR. The expression pattern of 41 genes encompassing multiple pathways integral to milk protein synthesis including insulin, mammalian target of rapamycin (mTOR), 5' AMP-activated protein kinase, Jak2-Stat5 signaling, and the expression of glucose and AA transporters was evaluated. Our results confirmed that most upregulated genes increased from $\mathrm{d}-15$ and peaked at d 30 or 60 and then remained relatively highly expressed. Specifically, there was an increased expression of mTOR-related amino acid transporters (SLC1A5, SLC7A5, and SLC36A1), glucose transporters (SLC2A1, SLC2A3, and SLC2A8), Jak2-Stat5 pathway $(E L F 5)$, and insulin signaling pathway components (IRS1, PDPK1, and AKT1). For activation of proteins synthesis, MTOR was significantly increased only at d 1. Among inhibitors of mTOR signaling, TSC1 and PRKAA2 were significantly upregulated during lactation. The RPL23 was downregulated among ribosomal components. In conclusion, a critical role for AA and glucose transporters and insulin signaling through mTOR for regulation of yak milk protein synthesis was revealed in this study of the yak mammary gland.

Received March 7, 2018.

Accepted August 6, 2018

${ }^{1}$ Corresponding author: Mingfengjiang@vip.sina.com
Key words: protein synthesis, yak mammary, mammalian target of rapamycin, amino acid and glucose transporters

\section{Short Communication}

The yak (Bos grunniens) lives throughout the Tibetan plateau in western China at high altitudes where hostile environmental factors exist including low humidity, temperature, and oxygen levels; strong winds; and UV radiation. The yak has adapted to thrive under these harsh conditions, where few other domestic animals could survive. The yak is a crucial resource to supply milk, meat, hair, and cheese to the people localized in Tibetan plateau area. For these reasons, the yak is one of the most critical domestic animals for the 6.5 million Tibetan people (Harris et al., 1999).

Based on the fact that the yak represents the primary source of milk for the people of this region, it is imperative to understand the genetic makeup of the yak and how this can influence milk biosynthesis. Yak milk has a high content of TS (16.9-17.9\%), protein (4.9-5.9\%), and fat (5.5-7.5\%; Jelen, 2000, Wiener et al., 2003). These values are significantly higher than those of cow (Bos taurus) and goat (Capra hircus) milk, but relatively similar to those of buffalo (Bubalus bubalis) and ewe (Ovis aries) milk (Jelen, 2000). Therefore, determining specific molecular signatures associated with milk synthesis in yaks is essential to potentially enhance milk quality and yield. Using data generated from high-producing dairy cows has limitations because yaks differ substantially from dairy cows. A key focus in the field of yak research is uncovering which genes control the production and composition of milk, how these genes are regulated, and how they might be manipulated to enhance milk yield, manufacturing properties, and health characteristics.

Over the last several decades, protein has become a highly valued constituent of dairy cow milk, and con- 


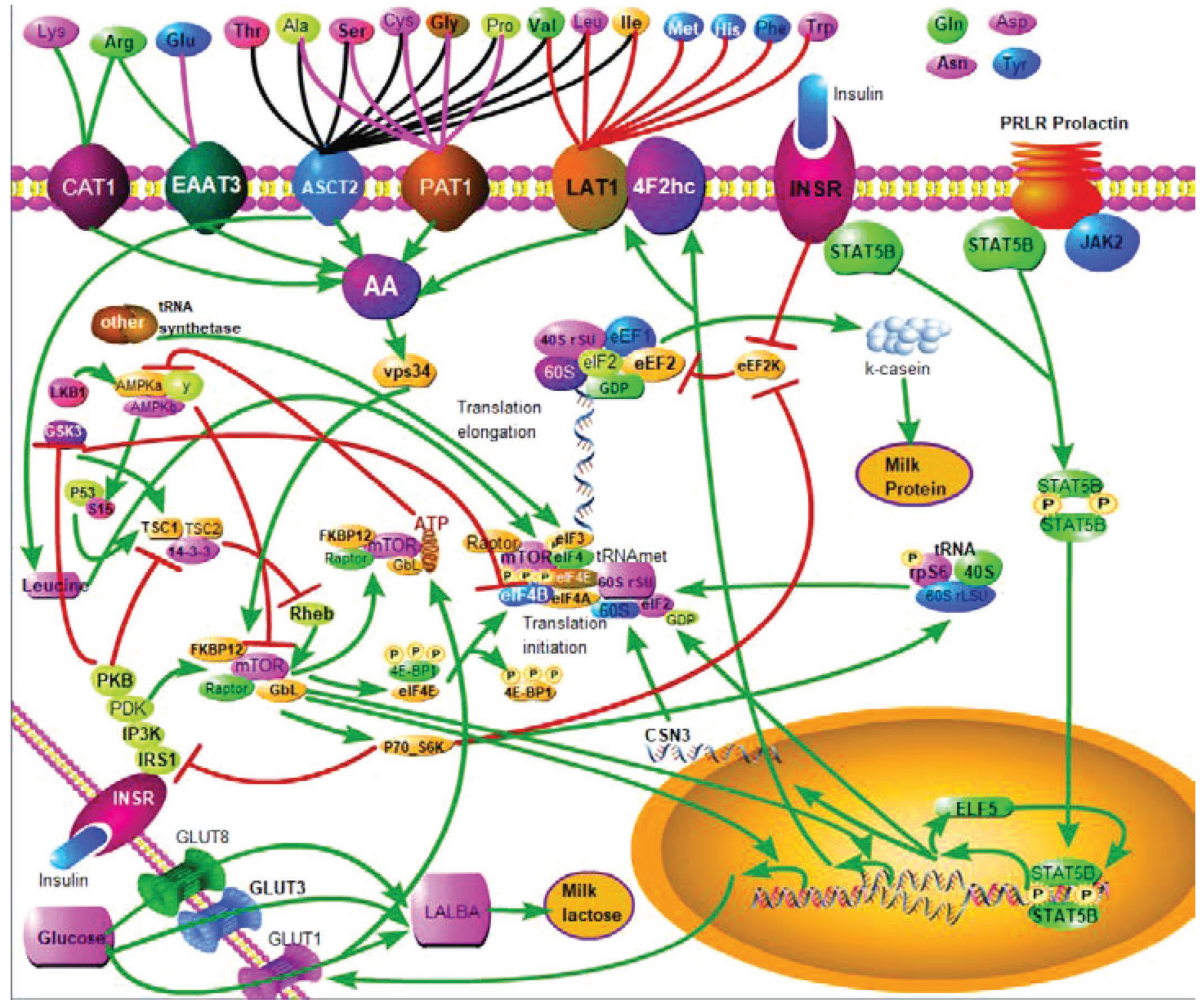

Figure 1. Networks of factors (genes product, hormones, and metabolites) potentially involved in the regulation of milk protein synthesis in lactating yak mammary tissue. The network includes the transport of AA and glucose, insulin signaling, JAK-STAT5 signaling, and activation/ inhibition of protein synthesis through the mammalian target of rapamycin (mTOR) pathway. * Denotes proteins where the gene expression was measured in the present experiment. The arrows denote activation $(\rightarrow)$ or inhibition $(-\mid)$. Color version available online.

sequently milk protein yield has gained a strong focus in modern breeding programs (Lipkin et al., 2008). Recent work demonstrated a pivotal role of insulin in regulating milk protein synthesis both in cows and mice (Menzies et al., 2009, 2010). The main regulator of milk protein expression in nonruminant mammary glands was found to be the Jak-Stat signaling pathway (Yang et al., 2000a,b). However, the regulation of protein synthesis, particularly translation, in all tissues of mammals appears to be under control of the mammalian target of rapamycin (mTOR) pathway (Wang and Proud, 2006). Therefore, according to previously pub- lished research elucidating the gene expression network of bovine milk protein gene synthesis (Bionaz and Loor, 2011) or studies of bovine mammary epithelial cells (Sigl et al., 2014), 41 genes with key roles in mTOR signaling, insulin signaling, AA transport, glucose transport, and Jak2-Stat5 signaling were selected in this study. A depiction of this network in yak mammary tissue and potential interactions is summarized in Figure 1.

To assess the role of the aforementioned genes in the mammary gland of yak, and specifically in milk protein synthesis, we biopsied yak mammary tissues during late pregnancy and subsequent lactation. 

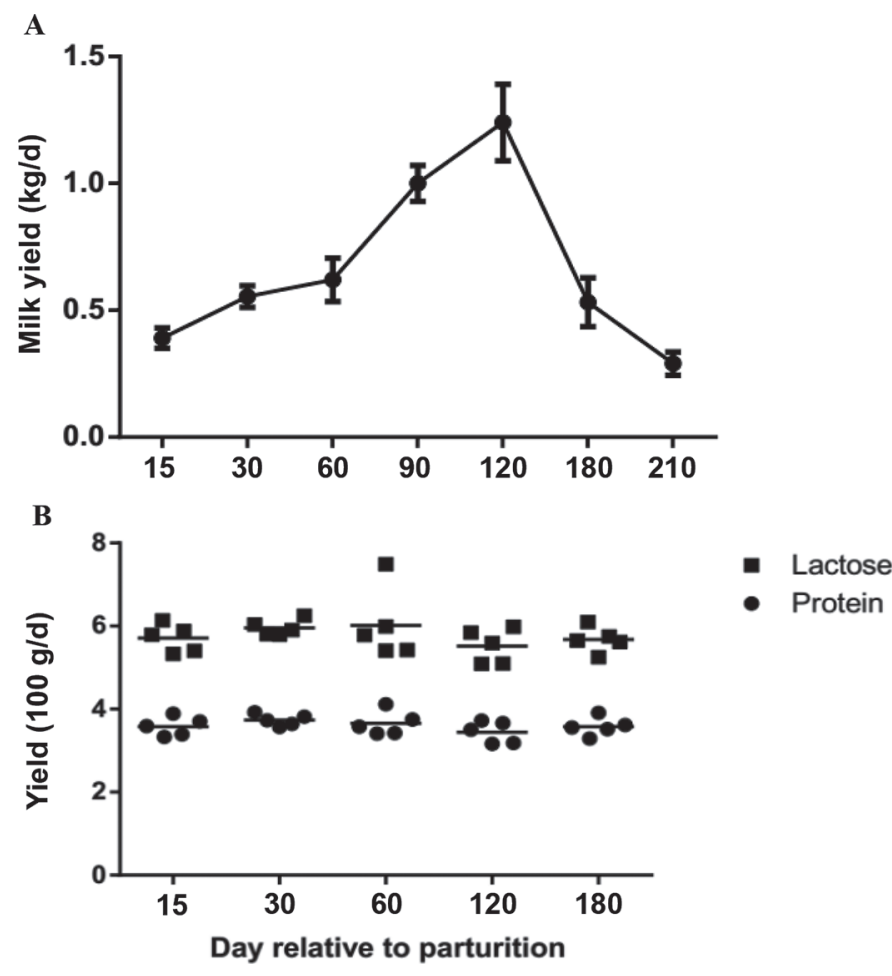

Figure 2. (A) Milk yields and (B) milk lactose and protein yield during lactation. Error bars represent SE.

Five female yaks of high genetic merit from Hongyuan County in the Sichuan Province in China were used in this study. Mammary tissue samples $(\sim 1 \mathrm{~g})$ were collected from the right or left rear quarters at $-15,1,15,30,60,120$, and $240 \mathrm{~d}$ relative to parturition as previously described (Bionaz and Loor, 2007; Lee et al., 2017). Milk yield was recorded during the corresponding biopsy days (Figure 2A) and has been previously published (Lee et al., 2017) with one additional yak. All procedures and protocols performed in this study were approved by the Southwest Minzu University Institutional Animal Care and Use Committee (permit number 2011-3-2).

The RNA extraction method was conducted as previously described (Bionaz and Loor, 2007). Briefly, yak mammary tissue was weighed and homogenized in TRIzol to extract RNA, and the purity was determined by UV/visible spectrophotometer (Eppendorf, Hamburg, Germany), and the integrity was assessed using $1 \%$ gel electrophoresis.

The genomic DNA removal procedure was the same as previously described (Bionaz and Loor, 2007). Briefly, RNA was diluted to $200 \mathrm{ng} / \mu \mathrm{L}$ using DNase and RNasefree water and $600 \mathrm{ng}$ of RNA was used for genomic DNA removal by PrimeScriptRT reagent kit with gDNA Eraser (Takara, Shiga, Japan). The cDNA was synthesized using PrimeScriptRT reagent kit (Takara) follow- ing the manufacturer's instructions (http://www.takara .com.cn/ProductShow.aspx? $\mathrm{m}=20141215102916640154$ \&productID $=20141226142531140921$ ). The kit included a blend of oligo-dT and random hexamer primers for reverse transcription. The synthesized cDNA was diluted 1:3 with DNase and RNase-free water before real-time quantitative PCR (RT-qPCR).

Genes related to milk protein synthesis for RT-qPCR were selected based on a previous study, and the primers were designed based on yak genes using Beacon Designer 7.6 software by fixing the amplicon size between 80 and $150 \mathrm{bp}$ and the melting temperature was between 55 and $75^{\circ} \mathrm{C}$ (Bionaz and Loor, 2011). The selected gene sequences were obtained from the yak reference genome (BosGru_v2.0) with BWA (v0.7.15; Qiu et al., 2012). The optimal annealing temperature of each primer set was determined by gradient PCR. The product of each primer pair was verified by electrophoresis analysis on a $2 \%$ agarose gel to check for amplicon size and the absence of primer-dimers. The specificity of the amplicon was also verified by the presence of a single peak during the dissociation curve after RT-qPCR cycling protocol. The amplicon for each primer pair was also purified, sequenced by a 3730 DNA analyzer (ABI, Waltham, MA) and the sequence was confirmed in the Basic Local Alignment Search Tool (BLAST) against all possible transcript sequences in the National Center for Biotechnology Information. The final PCR primer sequences are presented in Supplemental Table S1 (https://doi.org/10.3168/jds.2018-14715).

For each sample, the RT-qPCR was carried out in triplicates using the CFX96 Real-time system (BioRad, Hercules, CA). A 6-point standard curve was generated for each gene using a 10-fold dilution of cDNA to determine the efficiency of amplification for each primer pair. The RT-qPCR was performed in a $10 \mu \mathrm{L}$ system containing $2 \mu \mathrm{L}$ of diluted cDNA, $5 \mu \mathrm{L}$ of SsoFast EvaGreen supermix (Bio-Rad), 0.5 $\mu \mathrm{L}$ of each forward and reverse primers at $10 \mu \mathrm{M}$, and $2 \mu \mathrm{L}$ of DNase in RNase-free water. The instrument was set at $95^{\circ} \mathrm{C}$ for $10 \mathrm{~min}, 40$ cycles of $15 \mathrm{~s}$ at $95^{\circ} \mathrm{C}$, and 1 min at optimal annealing temperature between 55 and $\sim 63^{\circ} \mathrm{C}$ (Supplemental Table S1). The presence of a single PCR product was verified by the dissociation protocol using $95^{\circ} \mathrm{C}$ for $15 \mathrm{~s}, 65^{\circ} \mathrm{C}$ to $95^{\circ} \mathrm{C}$ for $15 \mathrm{~s}$. A negative control without cDNA template was included in each assay.

The reference genes MRPS15, RPS23, and UXT were used to normalize the RT-qPCR data, which were selected from 10 internal control genes (ACTB, EIF6, GAPDH, LRP10, MRPL39, MRPS15, MTG1, RPS8, RPS23, and UXT) using geNorm. Our normalization strategy for RT-qPCR data obtained from yak mammary tissue during pregnancy and lactation has been 
previously reported (Jiang et al., 2016). According to previous publications in our laboratory (Lee et al., 2017) and others (Bionaz and Loor, 2011), PCR-normalized data are presented as $\log _{2} \mathrm{n}$-fold change relative to $-15 \mathrm{~d}$. Then, to estimate standard errors at $-15 \mathrm{~d}$ and prevent biases in statistical analysis, normalized data were transformed to obtain a perfect average of 1.0 at $-15 \mathrm{~d}$, leaving the proportional difference between the biological replicates. The same proportional change was calculated at all other time points to obtain a fold change relative to $-15 \mathrm{~d}$. The data were $\log _{2}$ transformed before statistical analysis. The $\log _{2}$ transformed data relative to $-15 \mathrm{~d}$ were analyzed using the Proc MIXED of SAS with repeated measures (v9.4, SAS Institute Inc., Cary, NC) to evaluate the effect of time relative to parturition. Compound symmetry was used as covariate structure for repeated measures analysis. The model included the fixed time effect $(-15,1,15$, $30,60,120$, and $240 \mathrm{~d}$ ) and the random effect of yak. The data were discussed only if an overall time effect $(P$ $\leq 0.05)$ was present. Comparison between time points was declared significant at $P<0.05$.

The PCR amplification efficiency for each gene was calculated using the standard curve method $(\mathrm{E}=$ $10^{-1 /-\log \text { curve slope }}$. The mRNA abundance of measured genes was calculated as previously reported by others (Bionaz and Loor, 2011) and also in our laboratory (Lee et al., 2017), using the inverse of PCR efficiency raised to $\Delta \mathrm{Ct}$ (gene abundance $=1 / \mathrm{E}^{\Delta \mathrm{Ct}}$, where $\Delta \mathrm{Ct}=$ cycle threshold $(\mathbf{C t})$ sample - geometric mean $\mathrm{Ct}$ of 3 internal control genes). The mRNA abundance relative to $U X T$ for each transcript measured was calculated using the ratio of median $\Delta \mathrm{Ct}$ of the gene/median $\Delta \mathrm{Ct}$ of $U X T$. The latter is primarily done because relative mRNA quantification was performed using a standard curve and precluded a direct comparison among genes. Use of Ct values corrected for the efficiency of amplification plus internal control genes as baseline overcame this limitation. The description of genes measured and overall relative mRNA abundance relative to $U X T$ are shown in Table 1.

Gene expression patterns related to AA transporters, Jak2-Stat5 signaling, glucose transporters, ribosomal proteins, and caseins throughout the lactation in the yak mammary gland are presented in Figure 3. Expression of 3 intracellular AA transporters $(S L C 1 A 1, S L$ $C 1 A 5$, and $S L C 36 A 1)$ and 3 counter-transport cationic AA genes (SLC3A2, SLC7A1, and SLC7A5) in the yak mammary tissue are identified (Figure 3 ). Evidence of enhancement was revealed in most measured mTORrelated AA transporters such as $S L C 1 A 5, S L C 7 A 5$, and SLC36A1 during lactation relative to pregnancy at -15 $\mathrm{d}$ (Figure 3), where the relative mRNA abundance of $S L C 1 A 5$ and $S L C 7 A 5$ was evidently greater compared with other AA transporter genes (Table 1). In contrast to $S L C 1 A 5, S L C 7 A 5$, and $S L C 36 A 1$, a downregulation occurred in the expression of SLC3A2 during lactation. On the other hand, no significant difference was found in AA transporter genes unrelated to mTOR including $S L C 1 A 1$ and $S L C 7 A 1$. In addition, CSN3 was upregulated during lactation in the yak mammary gland (Figure 3), and its relative abundance was the highest among all the genes evaluated (Table 1). Interestingly, SLC3A2 was upregulated during bovine milk protein synthesis, although its expression was lower than other AAT, which is consistent in bovine and yak mammary gland (Bionaz and Loor, 2011). However, SLC7A1 expression was upregulated during lactation in bovine mammary gland (Bionaz and Loor, 2011). Amino acid availability is a limiting factor for milk protein synthesis (Baumrucker, 1985; Reynolds et al., 1994; Bequette et al., 1998; Rius et al., 2010); therefore, changes in gene expression of AAT can have profound effects in this process. Previous research also showed increased expression of $S L C 7 A 5$ in rodent milk protein synthesis (Alemán et al., 2009). The SLC1A5 encodes ASCT2, the major AA transporter, which transports the neutral AA Ala, Gly, Leu, Ile, Val, Ser, Cys, Thr, and Pro. The gene SLC7A5 codes for LAT1, which transports cationic AA. These 2 AAT have a regulatory relationship with $\mathrm{mTOR}$, and consequently control protein synthesis, cellular growth, and survival signaling, which might be related to cancer (Fuchs and Bode, 2005). It is reported that milk protein synthesis is regulated by T1R1/T1R3, a G protein-coupled taste receptor, through the mTOR pathway in the mouse mammary gland (Liu et al., 2017). The mTOR controls ribosome biogenesis and cell growth through several regulatory pathways. Research shows that the mTOR pathway is influenced by the intracellular concentration of ATP, independent of the abundance of AA, and that mTOR itself is an ATP sensor (Dennis et al., 2001). The SLC36A1 increased significantly in the yak mammary gland since $\mathrm{d} 1$ and throughout the lactation (Figure 3), which is also expressed in the small intestine (Thwaites and Anderson, 2007) and proven to regulate mTORC1 inside human breast cancer cells (Heublein et al., 2010). The effect of lactation in the yak mammary gland by upregulating several AAT and their potential control of mTOR signaling through the import of AA into cells underscores an essential role of AAT in yak mammary gland physiology. The expression of several AAT discussed above appears to be regulated by mTOR, suggesting the crucial importance of this pathway in milk protein synthesis, which has been demonstrated previously (Liu et al., 2017).

Expression patterns of 3 glucose transporters $S L$ C2A1, SLC2A3, and SLC2A8 were tested in the pres- 
ent study (Figure 3 ). These 3 glucose transporters were significantly upregulated from d 30 to 120 relative to pregnancy. Glucose is the primary precursor of lactose, the major solid component and osmolarity regulator in milk. Glucose uptake by the mammary glands increases dramatically to meet the requirements for milk synthesis (Zhao and Keating, 2007b), which is accomplished by increased expression of glucose transporters (GLUT). The increased expression of all 3 GLUT during lactation (Figure 3) is consistent with previous publications in the bovine mammary gland (Zhao and Keating, 2007a; Bionaz and Loor, 2011). Therefore, our results confirm the importance of glucose uptake by the yak mammary gland during lactation and enhanced expression of GLUT1, which also appears to be regulated by mTOR (Buller et al., 2008).

In the context of milk protein synthesis in the yak mammary gland, the unresponsive Jak2-Stat5 signal-

Table 1. Gene symbol, description, and mRNA abundance relative to UXT for the 41 genes measured; genes are clustered based on main functions relative to mammary protein synthesis

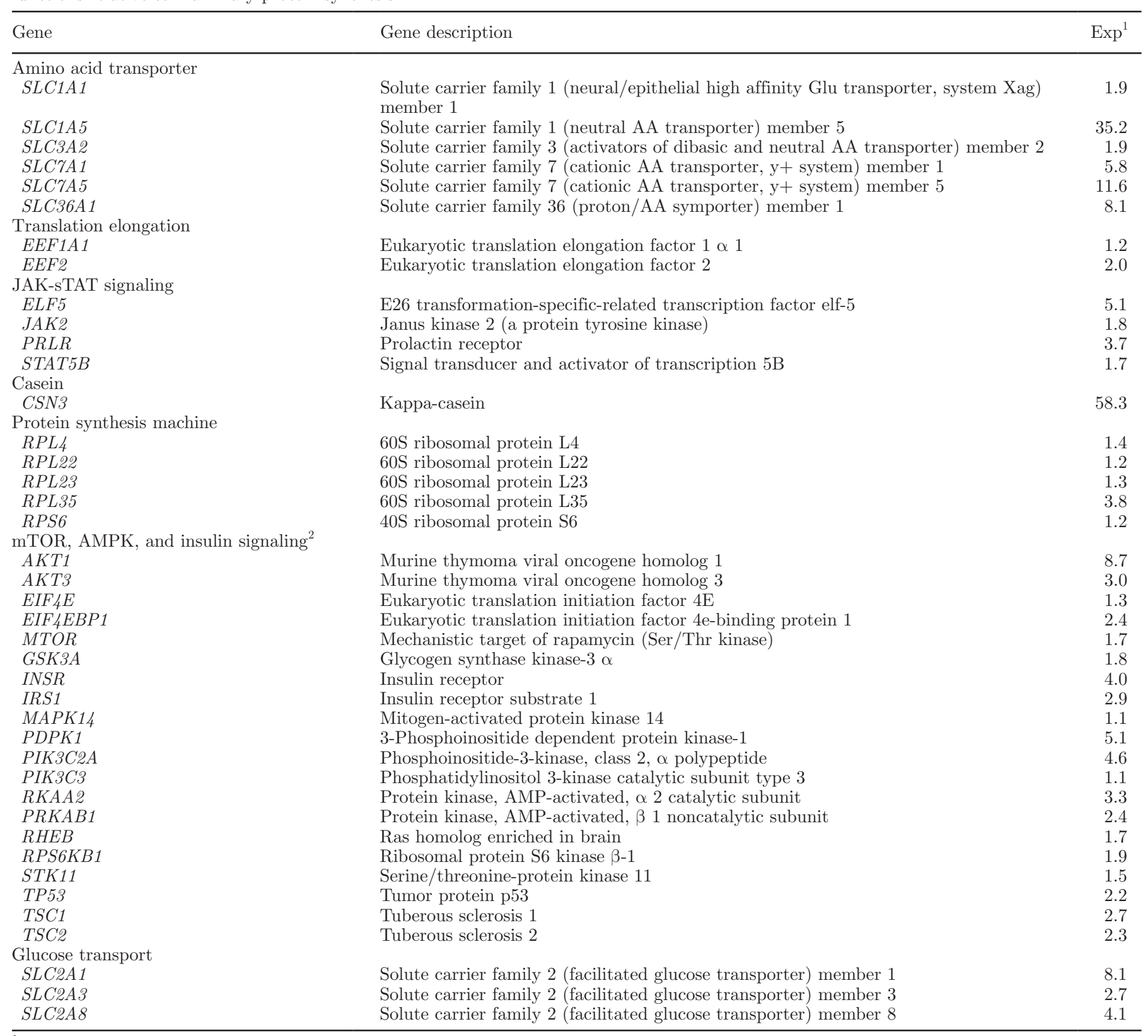

${ }^{1}$ mRNA abundance relative to $U X T$.

${ }^{2} \mathrm{mTOR}=$ mammalian target of rapamycin; AMPK $=5^{\prime}$ AMP-activated protein kinase. 
ing to changes over the lactation was supported by the lack of transcriptional changes in PRLR, JAKR, and STAT5B during lactation. Only ELF5 was upregulated from 1 to $60 \mathrm{~d}$ postpartum and at $240 \mathrm{~d}$ postpartum (Figure 3), which suggests a role of ELF5 in yak mam- mary protein synthesis. Prolactin signal transduction in mammary epithelial cells is mediated by a novel, direct signaling system that links the activation of the prolactin receptor at the cell surface level to changes in gene transcription in the nucleus. This pathway has been
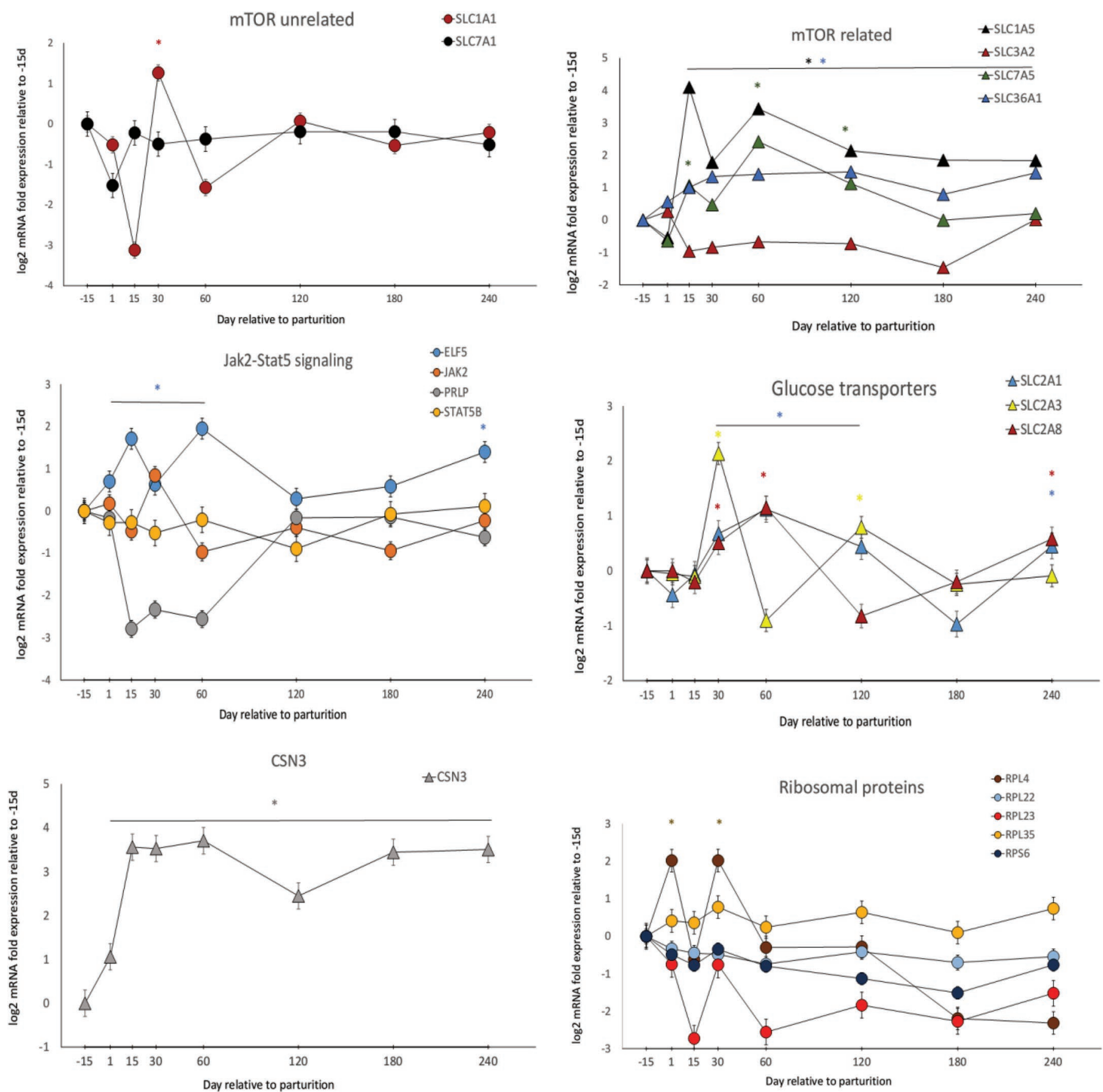

Figure 3. Change in expression during lactation relative to $-15 \mathrm{~d}$ of genes related to AA transport, glucose transport, ribosomal proteins, к-casein (CSN3), and Jak2-Stat5 signaling. The * denotes the time points significantly $(P<0.05)$ different than $-15 \mathrm{~d}$; each color of the * corresponds to the color of the symbol for the gene. $\mathrm{mTOR}=$ mammalian target of rapamycin; AMPK $=5^{\prime}$ AMP-activated protein kinase; CSN3 = kappa-casein. Error bars represent SE. Color version available online. 
identified as the Jak/Stat pathway, which is also used by many other growth factors and cytokines (Watson and Burdon, 1996). However, in yaks, a less important function for Jak2-Stat5 signaling in yak milk protein synthesis was revealed in our results, primarily due to the lack of change in expression of $P R L R, J A K 2$, and STAT5B during yak lactation. The ELF5 was signifi- cantly upregulated during lactation (Figure 3), which is similar with the result in bovine mammary gland (Bionaz and Loor, 2011), indicating a similar function of ELF5 in yak mammary protein synthesis. The ELF5 is reported to be essential for early embryogenesis and mammary gland development during pregnancy and lactation (Zhou et al., 2005). In fact, ELF5 has been
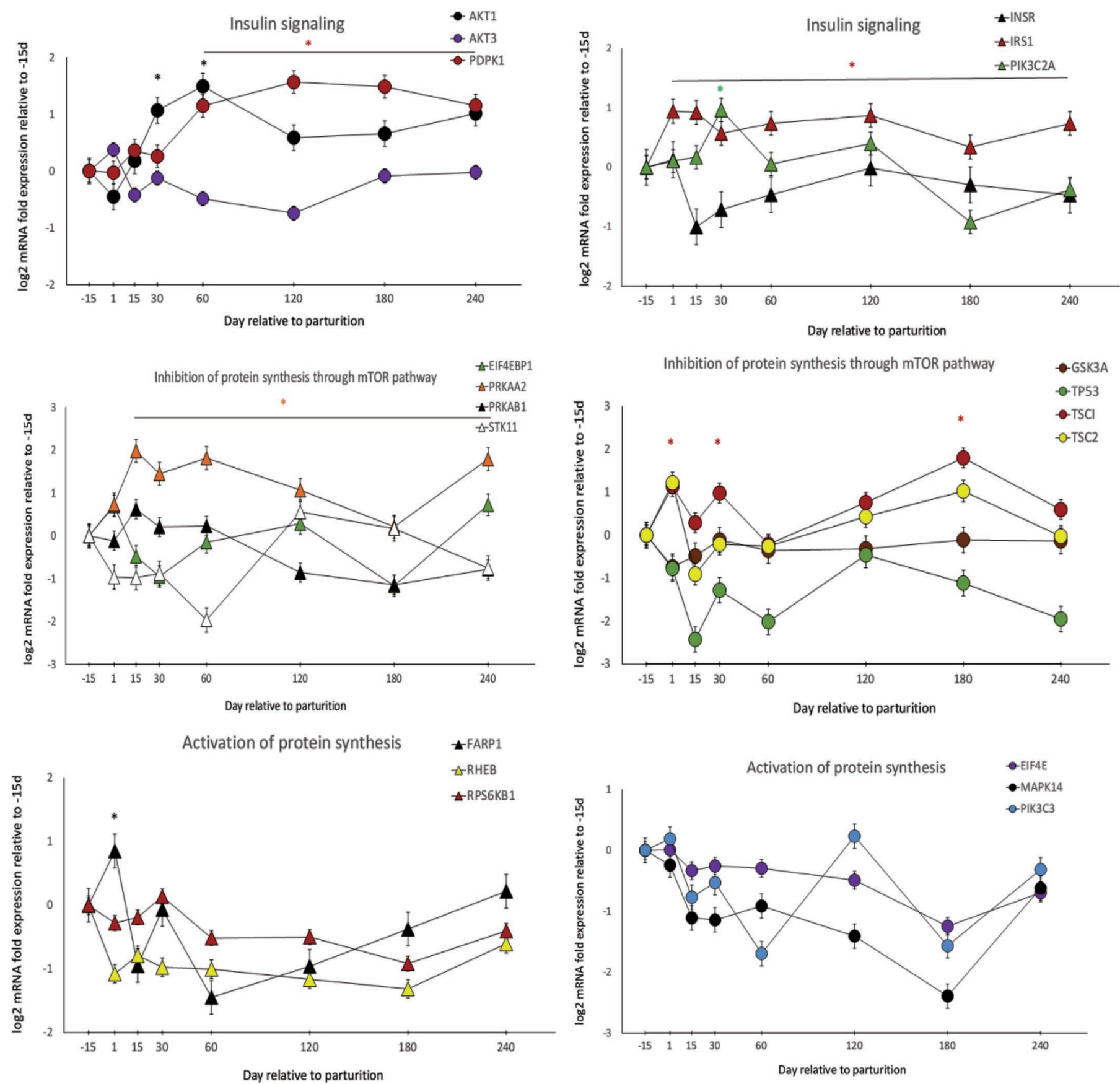

Figure 4. Change in expression during lactation relative to $-15 \mathrm{~d}$ of genes related to insulin signaling, activation, and inhibition of protein synthesis related to mammalian target of rapamycin $(\mathrm{mTOR})$. The ${ }^{*}$ denotes the time points significantly $(P<0.05)$ different than $-15 \mathrm{~d}$; each color of the * corresponds to the color of the symbol for the gene. Error bars represent SE. Color version available online. 
shown to be induced by insulin in other species (Kuraishi et al., 2000), and the beneficial effect of insulin in milk protein synthesis has been confirmed (Choi et al., 2004).

Gene expression patterns associated with insulin signaling, mTOR pathway, and protein synthesis throughout the lactation in yak mammary gland are presented in Figure 4. Significant upregulation was observed for IRS1, which is involved in intracellular signaling during lactation (Figure 4). The INSR remained unchanged during the lactation and PIK3C2A was upregulated only at $\mathrm{d} 30$. The mRNA expression of $P D P K 1$, a gene involved in insulin signaling downstream of IRS1, was upregulated from d 60 to 240 . The AKT1 expression was higher only at d 30 and 60 (Figure 4). The AKT3 remained unchanged during the lactation (Figure 4). In bovine mammary epithelial cells, it has been reported that leucine and histidine independently regulate milk protein synthesis via the mTOR signaling pathway (Gao et al., 2015). Moreover, a study in bovine also showed significantly upregulation in INSR (Bionaz and Loor, 2011). Downstream of IRS1 involved in insulin signaling, a significant upregulation was observed for $P D P K 1$ during the entire lactation, which is consistent with previous data in the bovine mammary gland (Bionaz and Loor, 2011). Signal transduction molecules $A K T 1$ and mTOR are essential for transcription and translation of genes related to milk protein synthesis in mammary epithelial cells (Shi et al., 2013; Chen et al., 2017). Furthermore, a study reported the upregulation of $A K T 1$ in sow mammary tissue from pregnancy to lactation and its effect on milk lactose synthesis (Chen et al., 2017). In the bovine, AKT3 was significantly increased (Bionaz and Loor, 2011).

For the activation of proteins synthesis, a significant increase was observed during lactation for MTOR only at d 1 (Figure 4). Among the downstream targets of mTOR, the expression of EIF4E, MAPK14, and PIK3C3 were did not change over time (Figure 4). Conversely, MTOR remained consistently increased in bovine (Bionaz and Loor, 2011). The mRNA expression of MAPK14 and EIF $4 E$ were upregulated during lactation in the bovine mammary gland; however, this effect was not observed in the current yak study.

Among inhibitors of mTOR signaling, TSC1 was upregulated during lactation at $\mathrm{d} 1,30$, and 180 (Figure 4 ), and PRKAA2 was upregulated during the entire lactation. The TSC1, inhibitor of mTOR signaling, was altered during the yak lactation, which is consistent with the results in bovine (Bionaz and Loor, 2011). However, the additional GSK3A also showed an increase in bovine milk synthesis (Bionaz and Loor, 2011), which was not changed in yaks.
For ribosomal proteins, RPL23 expression was downregulated during peak lactation (Figure 3), whereas only $R P L 4$ had a significant upregulation at $\mathrm{d} 1$ and 30 (Figure 3). Our results suggest that some aspects of protein synthesis process not only did not increase during lactation in the yak mammary gland, but also, in fact, decreased, which has been reported previously in bovine milk protein synthesis (Toerien et al., 2010). Decreased expression of protein synthesis components during lactation has also been reported in mice (Lemay et al., 2007).

Supplemental Figures S1, S2, and S3 (https://doi .org/10.3168/jds.2018-14715) summarize the dynamic networks of measured genes in mTOR, $5^{\prime}$ AMP-activated protein kinase, and insulin signaling. Overall, network visualization indicated that during lactation the expression of genes composing the networks is quite stable and with few changes. Overall, the genes SLC7A5, AMPK, PDK1, and IRS1 are constantly upregulated and $A K T$ changed into a highly expressed level. Essential AA transportation into cells through the SLC3A2/SLC7A5 heterodimer is considered ratelimiting for EAA activation of mTORC1 (Nicklin et al., 2009). In conclusion, our results demonstrated a critical role of cell transporters including mTOR-related AA and glucose as well as insulin signaling through mTOR for regulation of milk protein synthesis in the yak mammary gland.

\section{ACKNOWLEDGMENTS}

This work was supported financially by a grant from the Sichuan Youth Science and Technology Innovation Team (2015TD0025), The National Natural Science Foundation of China (31172198), and the National Sci-Tech Support Plan (2014BAD13B03). We appreciate the bioinformation analysis by Bingyu Yan from Purdue University (West Lafayette, IN).

\section{REFERENCES}

Alemán, G., A. López, G. Ordaz, N. Torres, and A. R. Tovar. 2009. Changes in messenger RNA abundance of amino acid transporters in rat mammary gland during pregnancy, lactation, and weaning. Metabolism 58:594-601.

Baumrucker, C. R. 1985. Amino acid transport systems in bovine mammary tissue. J. Dairy Sci. 68:2436-2451.

Bequette, B. J., F. R. Backwell, and L. A. Crompton. 1998. Current concepts of amino acid and protein metabolism in the mammary gland of the lactating ruminant. J. Dairy Sci. 81:2540-2559.

Bionaz, M., and J. J. Loor. 2007. Identification of reference genes for quantitative real-time PCR in the bovine mammary gland during the lactation cycle. Physiol. Genomics 29:312-319.

Bionaz, M., and J. J. Loor. 2011. Gene networks driving bovine mammary protein synthesis during the lactation cycle. Bioinform. Biol. Insights 5:83-98.

Buller, C. L., R. D. Loberg, M. H. Fan, Q. Zhu, J. L. Park, E. Vesely, K. Inoki, and K. L. Guan. 2008. A GSK-3/TSC2/mTOR pathway 
regulates glucose uptake and GLUT1 glucose transporter expression. Am. J. Physiol. Cell Physiol. 295:C836.

Chen, F., B. Chen, W. Guan, J. Chen, Y. Lv, H. Qiao, C. Wang, and Y. Zhang. 2017. Metabolic transition of milk lactose synthesis and up-regulation by AKT1 in sows from late pregnancy to lactation. Cell Biochem. Biophys. 75:131-138.

Choi, K. M., I. Barash, and R. E. Rhoads. 2004. Insulin and prolactin synergistically stimulate beta-casein messenger ribonucleic acid translation by cytoplasmic polyadenylation. Mol. Endocrinol. 18:1670.

Dennis, P. B., A. Jaeschke, M. Saitoh, B. Fowler, S. C. Kozma, and G. Thomas. 2001. Mammalian TOR: A homeostatic ATP sensor. Science 294:1102-1105.

Fuchs, B. C., and B. P. Bode. 2005. Amino acid transporters ASCT2 and LAT1 in cancer: Partners in crime? Semin. Cancer Biol. $15: 254-266$.

Gao, H. N., H. Hu, N. Zheng, and J. Q. Wang. 2015. Leucine and histidine independently regulate milk protein synthesis in bovine mammary epithelial cells via mTOR signaling pathway. J. Zhejiang Univ. Sci. B 16:560.

Harris, R. B., D. H. Pletscher, C. O. Loggers, and D. J. Miller. 1999 Status and trends of Tibetan plateau mammalian fauna, Yeniugou, China. Biol. Conserv. 87:13-19.

Heublein, S., S. Kazi, M. H. Ögmundsdóttir, E. V. Attwood, S. Kala C. A. R. Boyd, C. Wilson, and D. C. I. Goberdhan. 2010. Protonassisted amino acid transporters are conserved regulators of proliferation and amino acid-dependent mTORC1 activation. Oncogene 29:4068.

Jelen, P. 2000. Dairy Technology: P. Walstra, T. J. Geurts, A. Noomen, A. Jellema and M. A. J. S. van Boekel, Marcel Dekker, New York, 1999. pp. XVII+727, price US $\$ 225$, ISBN 0-8247-0228-X. Int. Dairy J. 10:585-586.

Jiang, M., J. N. Lee, M. Bionaz, X. Y. Deng, and Y. Wang. 2016. Evaluation of suitable internal control genes for RT-qPCR in yak mammary tissue during the lactation cycle. PLoS One 11:e0147705.

Kuraishi, T., F. I. K. Aoki, S. Sakai, and Y. Sun. 2000. The poly(A) tail length of casein mRNA in the lactating mammary gland changes depending upon the accumulation and removal of milk. Biochem. J. 347:579-583.

Lee, J. N., Y. Wang, Y. O. Xu, Y. C. Li, F. Tian, and M. F. Jiang. 2017. Characterisation of gene expression related to milk fat synthesis in the mammary tissue of lactating yaks. J. Dairy Res. $84: 283-288$.

Lemay, D. G., M. C. Neville, M. C. Rudolph, K. S. Pollard, and J. German. 2007. Gene regulatory networks in lactation: Identification of global principles using bioinformatics. BMC Syst. Biol. $1: 56$.

Lipkin, E., A. Bagnato, and M. Soller. 2008. Expected effects on protein yield of marker-assisted selection at quantitative trait loci affecting milk yield and milk protein percentage. J. Dairy Sci. 91:2857.

Liu, J., Y. Wang, D. Li, M. Li, C. Chen, X. Fang, H. Chen, and C. Zhang. 2017. Milk protein synthesis is regulated by T1R1/T1R3, a $\mathrm{G}$ protein-coupled taste receptor, through the mTOR pathway in the mouse mammary gland. Mol. Nutr. Food Res. 61. https://doi .org/10.1002/mnfr.201601017.

Menzies, K. K., H. J. Lee, C. Lefèvre, C. J. Ormandy, K. L. Macmillan, and K. R. Nicholas. 2010. Insulin, a key regulator of hormone responsive milk protein synthesis during lactogenesis in murine mammary explants. Funct. Integr. Genomics 10:87-95.
Menzies, K. K., C. Lefèvre, K. L. Macmillan, and K. R. Nicholas. 2009 Insulin regulates milk protein synthesis at multiple levels in the bovine mammary gland. Funct. Integr. Genomics 9:197.

Nicklin, P., P. Bergman, B. Zhang, E. Triantafellow, H. Wang, B. Nyfeler, H. Yang, M. Hild, C. Kung, and C. Wilson. 2009. Bidirectional transport of amino acids regulates mTOR and autophagy. Cell 136:521-534.

Qiu, Q., G. Zhang, T. Ma, W. Qian, J. Wang, Z. Ye, C. Cao, Q. Hu, J. Kim, and D. M. Larkin. 2012. The yak genome and adaptation to life at high altitude. Nat. Genet. 44:946-949.

Reynolds, C. K. D. L. Harmon, and M. J. Cecava. 1994. Absorption and delivery of nutrients for milk protein synthesis by portaldrained viscera. J. Dairy Sci. 77:2787-2808.

Rius, A. G., J. A. D. R. N. Appuhamy, J. Cyriac, D. Kirovski, O Becvar, J. Escobar, M. L. Mcgilliard, B. J. Bequette, R. M. Akers, and M. D. Hanigan. 2010. Regulation of protein synthesis in mammary glands of lactating dairy cows by starch and amino acids. J. Dairy Sci. 93:3114-3127.

Shi, L. L., F. Zhao, X. J. Gao, B. I. Wei-Wei, W. U. Cai-Xia, and L. I. Qing-Zhang. 2013. Effection of DIP and IGF-I on the expression of AKT-1 and mTOR genes in bovine mammary epithelial cells. China Anim. Husbandry Vet. Med. 40:23-27.

Sigl, T., H. H. Meyer, and S. Wiedemann. 2014. Gene expression analysis of protein synthesis pathways in bovine mammary epithelial cells purified from milk during lactation and short-term restricted feeding. J. Anim. Physiol. Anim. Nutr. (Berl.) 98:84-95.

Thwaites, D. T., and C. M. H. Anderson. 2007. H + -coupled nutrient, micronutrient and drug transporters in the mammalian small intestine. Exp. Physiol. 92:603-619.

Toerien, C. A., D. R. Trout, and J. P. Cant. 2010. Nutritional stimulation of milk protein yield of cows is associated with changes in phosphorylation of mammary eukaryotic initiation factor 2 and ribosomal s6 kinase 1. J. Nutr. 140:285-292.

Wang, X., and C. G. Proud. 2006. The mTOR pathway in the control of protein synthesis. Physiology (Bethesda) 21:362.

Watson, C. J., and T. G. Burdon. 1996. Prolactin signal transduction mechanisms in the mammary gland: the role of the Jak/Stat pathway. Rev. Reprod. 1:1-5.

Wiener, G., J. L. Han, and R. J. Long. 2003. Production Characteristics of Yak. FAO Regional Office for Asia and the Pacific, Bangkok, Thailand.

Yang, J., J. J. Kennelly, and V. E. Baracos. 2000a. The activity of transcription factor Stat5 responds to prolactin, growth hormone, and IGF-I in rat and bovine mammary explant culture. J. Anim. Sci. 78:3114-3125.

Yang, J., J. J. Kennelly, and V. E. Baracos. 2000b. Physiological levels of Stat5 DNA binding activity and protein in bovine mammary gland. J. Anim. Sci. 78:3126-3134.

Zhao, F. Q., and A. F. Keating. 2007a. Expression and regulation of glucose transporters in the bovine mammary gland. J. Dairy Sci. 90(Suppl 1):E76-E86.

Zhao, F. Q., and A. F. Keating. 2007b. Functional properties and genomics of glucose transporters. Curr. Genomics 8:113.

Zhou, J., R. Chehab, J. Tkalcevic, M. J. Naylor, J. Harris, T. J. Wilson, S. Tsao, I. Tellis, S. Zavarsek, and D. Xu. 2005. Elf5 is essential for early embryogenesis and mammary gland development during pregnancy and lactation. EMBO J. 24:635-644. 